\title{
Responsabilidad social universitaria en dos facultades de la Universidad Nacional del Centro del Perú-2019
}

\author{
Diógenes Orlando Hurtado Tiza \\ hytod@ hotmail.com \\ Rosa María Torres Huamancaja \\ Romath0119@gmail.com \\ William Armando Reyes Alva \\ villyreyes@gmail.com
}

\section{RESUMEN}

El objetivo general fue: Identificar la diferencia que existe entre la percepción que tienen los docentes de las facultades de Arquitectura e Ingeniería de Sistemas sobre el nivel de gestión de responsabilidad social universitaria en la Universidad Nacional del Centro del Perú. La metodología empleada fue cuantitativa, la tipología seguida es según lo estable Landeau (2007), básica, descriptiva, cuantitativa, transversa y de comprobación. Responde al diseño descriptivo comparativo, con una muestra censal de 68 docentes de ambas facultades de la UNCP, a quienes se aplicaron el cuestionario de gestión de responsabilidad social universitaria (adaptada Pro Calidad y Vallaeys), que fue validad por juicio de expertos (4) y la fiabilidad se hizo mediante prueba piloto obteniendo 0,960 según alfa de Cronbach. Se arribó la siguiente conclusión: Se identificó que existe diferencia significativa entre la percepción que tienen los docentes de las facultades de Arquitectura e Ingeniería de Sistemas sobre el nivel de responsabilidad social universitaria en la UNCP, Huancayo - 2019, con un nivel de significancia según U de Mann Whitney de 591.

Palabras clave: Gestión; formación; investigación; participación; social. 


\title{
University social responsibility in two faculties of the National University of the Center of Peru-2019
}

\begin{abstract}
:
The general objective was to: Identify the difference that exists between the teachers' perception of the faculties of Architecture and Systems Engineering on the level of university social responsibility management at the National University of Central Peru. The methodology used was quantitative, the typology followed is as stable Landeau (2007), basic, descriptive, quantitative, transverse and verification. It responds to the comparative descriptive design, with a census sample of 68 teachers from both faculties of the UNCP, to whom the questionnaire on university social responsibility management (adapted Pro Calidad and Vallaeys) was applied, which was valid by expert judgment (4) and the reliability was made by pilot test obtaining 0.960 according to Cronbach's alpha. The following conclusion was reached: It was identified that there is a significant difference between the teachers' perception of the faculties of Architecture and Systems Engineering on the level of university social responsibility in the UNCP, Huancayo 2019, with a significance level according to Mann Whitney's U of 591.
\end{abstract}

Keywords: Management; training; investigation; participation; social.

Artículo recibido: 01. agosto. 2020 Aceptado para publicación: 12. setiembre. 2020 Correspondenc hytod@hotmail.com Conflictos de Interés: Ninguna que declarar 


\section{INTRODUCCIÓN}

Las instituciones u organizaciones basan sus acciones y decisiones en instrumentos de gestión, en este caso al ser la UNCP una organización que es parte del Estado, se le caracteriza como una institución Pública, por lo que requiere de instrumentos de gestión pública para la toma de sus decisiones y logro de sus objetivos. las secuelas de su accionar sobre el desarrollo sustentable que se fundamentada en el crecimiento económico para todos, con políticas sociales incluyentes y equitativas, y así fomentar una armonía entre el medio ambiente, los actores sociales e instituciones (Naciones Unidas, 2005).

Según (Universidad Construye País, 2002,a) se encuentran preocupados con el nuevo escenario filosófico, político, ambiental y social de gestión que va exigiendo la sociedad en pleno en cuanto a su rol, funciones y compromisos éticos, social, económico y medio ambiental, denominándose a ella responsabilidad social universitaria (RSU). Los primeros estudios sobre ella surgen en Chile bajo la premisa de buscar la percepción de RSU según los stakeholders internos de la Universidad de Telemuco y con metodologías diversas. Por su parte, (Vallaeys, 2008) menciona que en las ultimas decadas recien se estan priorizando la responsabilidad social en las univeresidades publicas y privadas de todo el mundo.

Mientras que en el Perú sobresale de manera particular el Instituto de Responsabilidad Social (2003) y la Universidad del Pacífico, por su empeño en la difusión que realizan más de una década; también cabe destacar alguna empresa privada peruana realizando charlas en pro de la mejora de la calidad de los ciudadanos de los conos de la ciudad de Lima. Actualmente, muchas universidades difunden y practican la responsabilidad social universitaria, tal es el caso de la Universidad Nacional de Ingeniería, Universidad Mayor de San Marcos y de manera especial la Universidad San Agustín de Arequipa. A través del Sistema Nacional de Evaluación, Acreditación y Certificación de la Calidad Educativa (SINEACE) y del Consejo de Evaluación, Acreditación y Certificación de la Calidad de la Educación Superior Universitaria (CONEAU), dieron el inicio al proceso de sensibilización concerniente a la autoevaluación como parte de la acreditación en las universidades públicas y privadas, bajo la mirada de mejora en la gestión de la carrera, formación académica, 
investigación y servicios de apoyo o extensión universitaria. El Perú es la primer país en el mudo que ha creado una ley específicamente para la responsabilidad social universitaria en la Ley Universitaria $\mathrm{N}^{\circ}$ 30220, en su capítulo XIII, artículos 124 y 125, señala que la tarea de la RSU es la gestión ética y eficaz, enmarcada en la contribución y desarrollo sostenible del bienestar común de todos los actores sociales, en los ejes de gestión (cuatro), para ello la Ley revela de debe determinar el 2\% de su presupuesto a la RSU SINEACE (2016).

El Sistema de Responsabilidad Social Universitaria de la Universidad Nacional del Centro del Perú, específicamente nos referimos al objetivo principal de mitigar o reducir los impactos que la sociedad global genera y hace inviable a la misma sociedad global. Para ello, se caracteriza el desarrollo sostenible, en base al cual se identifica las causas y sus impactos que está generando la sociedad global y que hace inviable su sostenibilidad, el análisis se realiza en las cuatro dimensiones que comprende el desarrollo sostenible que son: ambiental, económico, social y político. La Nueva Gestión Pública desde una perspectiva neo pública; el cual se complementa con el enfoque y principios de la gestión basado en procesos identificando los procesos de nivel, como son: estratégico, de soporte y, misional o clave. La UNCP está obligada de cumplir, la interrelacionalidad, la transversalidad de los objetivos de la RSU con las funciones de la UNCP se hace imprescindible, lo cual obliga a integrar y cumplir los criterios de la RSU en las funciones de la UNCP, en los mismos procesos simultáneamente.

\section{MATERIALES Y MÉTODOS}

La presente investigación se encuadrada bajo el paradigma positivista, denominada como cuantitativa, empírico-analítico y racionalista, que busca describir, explicar, predecir, descubrir y controlar los fenómenos del mundo natural, utilizando la vía hipotético-deductivo (Tamayo, 1995). El tipo y diseño de investigación fue básica, descriptiva, cuantitativa, transversal y de comprobación (Landeau, 2007) y en concordancia a (Sierra, 2004) y (Hernández, et. al., 2014). La muestra fue censal de 68 docentes (FARQ (40) y FIS (28)), se aplicaron un cuestionario de 60 preguntas 
relacionados a gestión de RSU, la cual fue validad por cuatro expertos y la confiablidad fue de 0,96 según alfa de Cronbach. Se utilizó el método de análisis cuantitativo con estadígrafos descriptivos e inferenciales para los resultados y el método de contraste para la discusión. Finalmente, se consideraron los aspectos éticos, ya que el quehacer de las actitudes del científico, es de buscar la verdad con el sinceramiento de información, datos, metodología científica y el compromiso de buscar las explicaciones teóricas o prácticas en la solución de problemas de su entorno. Esta actitud es prospectiva, en marcada en los lineamientos éticos de la investigación, institucional y profesional (Koepsell, 2015).

\section{RESULTADOS Y DISCUSIÓN}

\section{Resultados de la variable gestión de responsabilidad social universitaria}

Objetivo general: planteada busca identificar la diferencia que existe entre la percepción de tienen los docentes de las facultades de Arquitectura e Ingeniería de Sistemas sobre el nivel de responsabilidad social universitaria en la UNCP, Huancayo -2019 .

Gráfico 1: Nivel de responsabilidad social universitaria

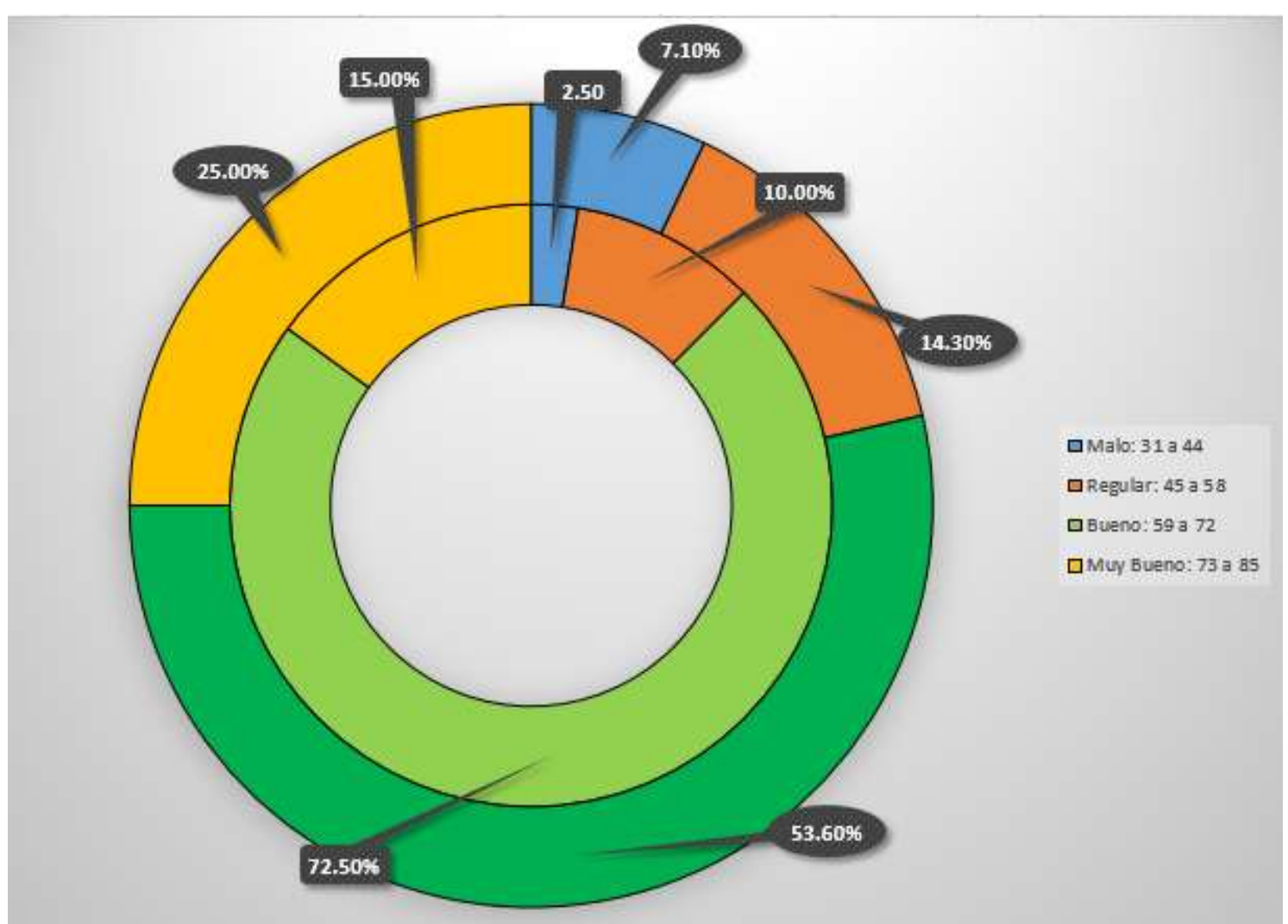

Fuente: Elaboración propia 
Se aprecia los resultados de la variable responsabilidad social universitaria. En la Facultad de Arquitectura, hay un docente que opina que esta dimensión se encuentra en el nivel malo (2.5\%). Mientras que hay 4 docentes (10\%) opina que ésta se encuentra en el nivel regular. De la misma manera hay 29 docentes $(72.5 \%)$ opinan que dicha dimensión se encuentra ubicada en el nivel bueno. Finalmente, se observa que 6 docentes (15\%) manifiesta que en la variable de responsabilidad social universitaria es muy bueno. En la Facultad de Ingeniería de Sistemas, hay dos docentes que opina que esta dimensión se encuentra en el nivel malo (7.1\%). Mientras que hay 4 docentes (14.3\%) opina que ésta se encuentra en el nivel regular. De la misma manera hay 15 docentes (53.6\%) opinan que dicha dimensión se encuentra ubicada en el nivel bueno. Finalmente, se observa que 7 docentes (25\%) manifiesta que en la variable de responsabilidad social universitaria es muy bueno.

Gráfico 2: Resultados de U Mann Whitney de responsabilidad social universitaria

\begin{tabular}{|c|c|c|c|c|}
\hline \multicolumn{5}{|c|}{ Resumen de prueba de hipótesis } \\
\hline & Hipótesis nula & Prueba & Sig. & Decisión \\
\hline 1 & $\begin{array}{l}\text { La distribución de PUNTAUE DE } \\
\text { RESPONSABILIDAD SOCIAL } \\
\text { UNIVERSITARIA es la misma entre } \\
\text { las categorias de FACULTADES. }\end{array}$ & $\begin{array}{l}\text { Prueba U de } \\
\text { Mann-Whitney } \\
\text { para muestras } \\
\text { independientes }\end{array}$ & 695,000 & $\begin{array}{l}\text { Retener la } \\
\text { hipotesis } \\
\text { nula. }\end{array}$ \\
\hline
\end{tabular}




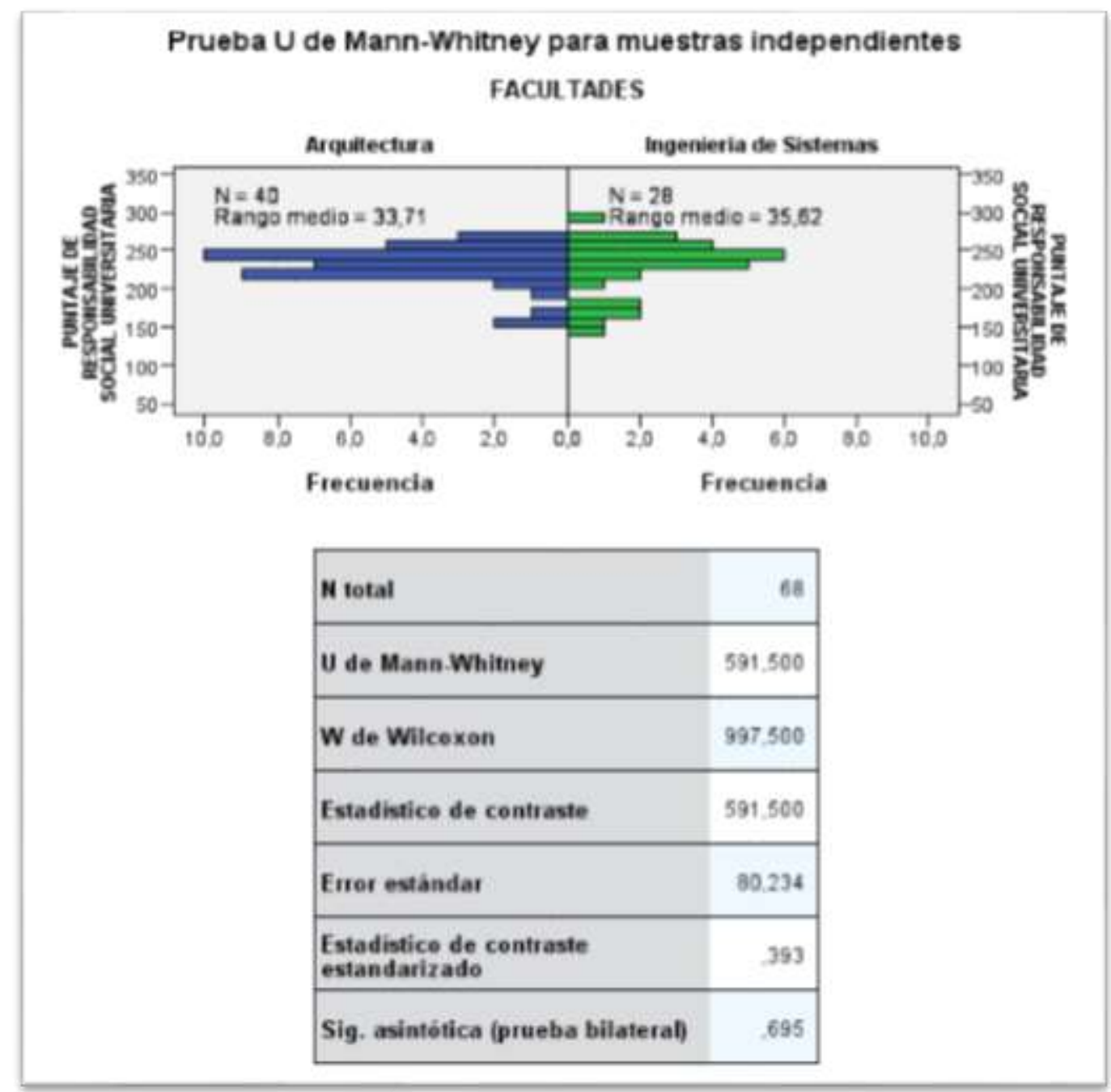

Fuente: Elaboración propia

\section{Decisión estadística:}

Conclusión estadística: Existe evidencias estadísticamente sustentables que existe diferencia significativa ente la percepción que tienen los docentes de las facultades de Arquitectura e Ingeniería de Sistemas sobre el nivel de responsabilidad social universitaria en la UNCP, Huancayo - 2019. Donde U de Mann Whitney de 695. 
Gráfico 3: Resultados de los objetivos específicos de la gestión de responsabilidad social universitaria

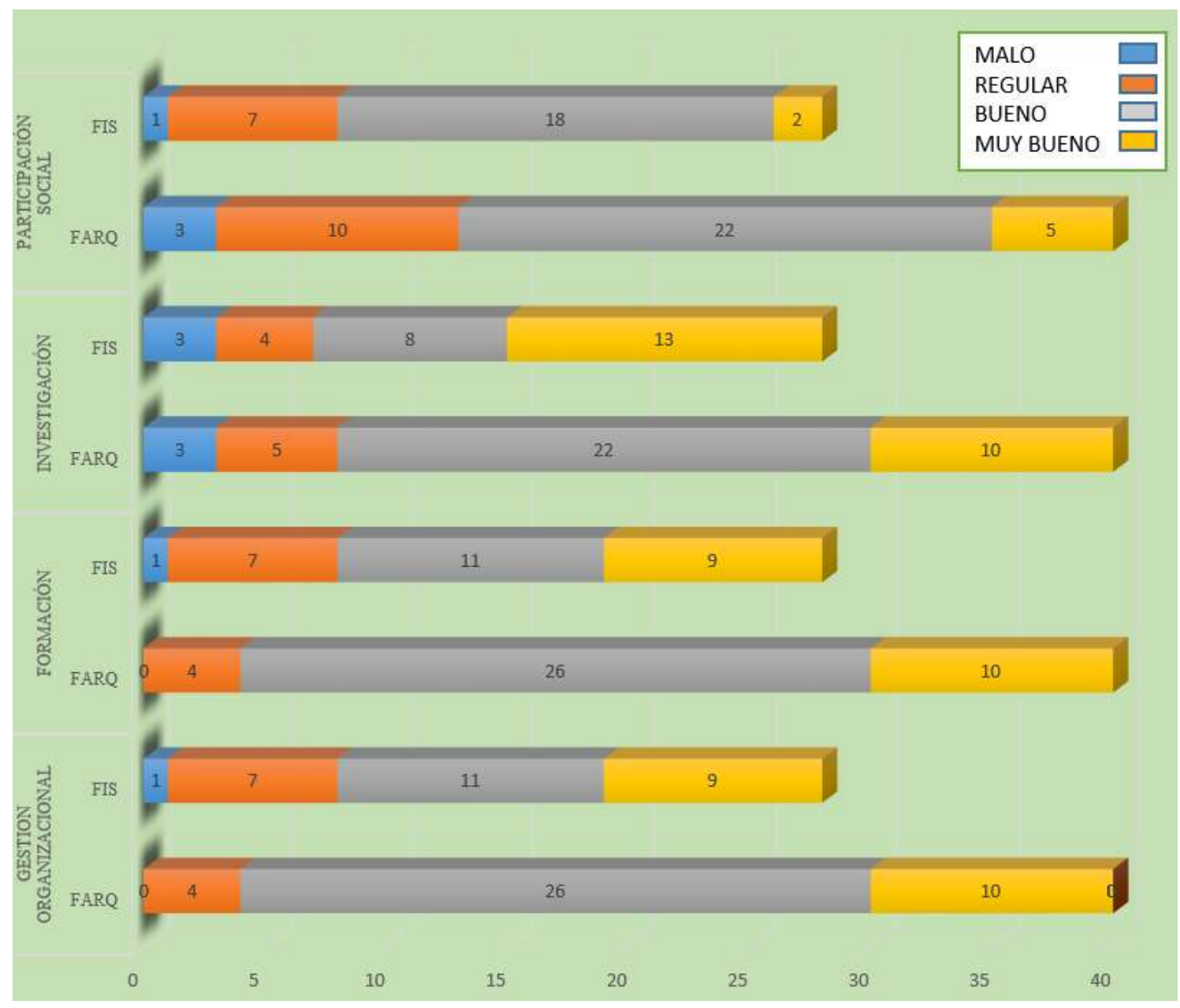

Fuente: Elaboración propia

En los resultados que se aprecia sobre las dimensiones: Gestión Organizacional, Formación, Investigación y Participación Social. En la Facultad de Arquitectura, hay cuatro, cinco y diez docentes que opina que se encuentra en el nivel Regular respectivamente. Mientras que hay 26, 65, 55, 22 docentes opinan que ésta se encuentra en el nivel bueno respectivamente.

En cuanto a la Facultad de Ingeniería de Sistemas en las dimensiones mencionadas; hay 7, 25 y 4 docentes que opina que se encuentra en el nivel Regular respectivamente. Mientras que hay 11, 8 y 18 docentes opinan que ésta se encuentra en el nivel bueno respectivamente. 
La intención de la investigación fue; los resultados encontrados en la investigación han comprobado que existe diferencia significativa entre la percepción que tienen los docentes de las facultades de Arquitectura e Ingeniería de Sistemas sobre el nivel de responsabilidad social universitaria en UNCP, Huancayo - 2019. Entre la percepción de los docentes de ambas facultades sobre la responsabilidad social universitaria es significativa según la U de Mann Whitney es 695.

Estas deducciones tienen proporción con lo que sostienen Baca (2016), Gil (2013), Gaete (2012), mencionan que la RSU en cuanto al alcance social que genera impacto entre las organizaciones, a la vez los programas sociales; en cuanto Alférez (2014), Vargas (2017) y Arispe (2016) en sus estudios de RSU buscan la percepción de la realidad y la responsabilidad que éstas mencionan; por último Flores (2015) y Reyna (2018) en sus estudios manifiestan que la gestión de la responsabilidad universitaria trascienden de manera significativa tanto de a la calidad académica como visto por las autoridades universitarias.

Mientras que los resultados por dimensiones los docentes de ambas facultades de acuerdo a que una de ellas se encuentra acreditada y la otra no; pero resultado refleja que ambas facultades su labor de responsabilidad social universitaria es buena. La exploración que contrastan dicho resultados, como el de buscar antecedentes que hablan sobe ello. (Alférez, 2014), (Baca, 2015), quienes encontraron que la forma como perciben los estudiantes/ administrativos y docentes varían de acuerdo a la experiencia que estas las tienen y todos ellos emplearon metodología cuantitativa para medir la RSU. Se debe mayormente a que la facultad no acredita su labor es netamente de trabajo con la sociedad; su responsabilidad lo asumen como tal, por la misma practica que comparten experiencias. Se conocen los procedimientos donde la responsabilidad social universitaria tanto estudiantes, como personal no docente y docentes, estas integran operaciones de aptitudes inherentes en el ser humano; lo contradictorio con la investigación, en el sentido, que ellos emplearon un instrumento que solo se aprecian los principios y valores de la RSU y no tanto lo que (Vallaeys F. , 2008) y (Flores, 2015) manifiestan que la RSU es una política filosófica que mide o aprecia la gestión de RSU en su cuatro componentes o dimensiones como es la gestión 
organizacional, formación, investigación y participación social. Lo que se desea es que se haga el efecto multiplicador en todas las universidades que aplique la ley universitaria en los artículos 124 y 125.

\section{CONCLUSIÓN O CONSIDERACIONES FINALES}

Se identificó que existe diferencia significativa entre la percepción que tienen los docentes de las facultades de Arquitectura e Ingeniería de Sistemas sobre el nivel de responsabilidad social universitaria en la UNCP, Huancayo - 2019, con un nivel de significancia de U de Mann Whitney de 591.

Se identificó que existe diferencia significativa entre la percepción que tienen los docentes de las facultades de Arquitectura e Ingeniería de Sistemas sobre la gestión organizacional en la UNCP, Huancayo - 2019, con un nivel de significancia de U de Mann Whitney de 528.

Se identificó que existe diferencia significativa entre la percepción que tienen los docentes de las facultades de Arquitectura e Ingeniería de Sistemas sobre el nivel de formación en la UNCP, Huancayo - 2019, con un nivel de significancia de U de Mann Whitney de 559.

Se identificó que existe diferencia significativa entre la percepción que tienen los docentes de las facultades de Arquitectura e Ingeniería de Sistemas sobre el nivel de investigación en la UNCP, Huancayo - 2019, con un nivel de significancia de U de Mann Whitney de 641.

Se identificó que existe diferencia significativa entre la percepción que tienen los docentes de las facultades de Arquitectura e Ingeniería de Sistemas sobre el nivel de participación social en la UNCP, Huancayo - 2019, con un nivel de significancia de U de Mann Whitney de 551. 


\section{LISTA DE REFERENCIAS}

Abanto, W. (2016). Diseño y desarrollo del proyecto de investigación: Guia de aprendizaje Doctorado. Trujillo-Perú: UPG "César Vallejo".

Alférez, A. (2014). Responsabilidad social universitaria en la formación curricular y la participación social: El Caso de la Facultad de Educación de la Universitat Intenacional de Catalunya. Barcelona-España: Tesis de grado. Universitat Internacional de Catalunya.

Arispe, E. (2016). La responsabilidad social universitaria y su relación con la interculturalidad en estudiantes universitarios, 2016. Lima: Tesis Maestría. Universidad de San Martin de Porres.

Baca, H. (2015). La responsabilidad social universitaria: propuesta conceptual y medición en el ámbito de una universidad privada de Lima-Perú. Sevilla - España: Tesis doctoral. Universidad de Sevilla.

Baca, H. (2016). La responsabilidad social universitaria: propuesta conceptual y medición en el ámbito de una universidad privada de Lima, Perú. Lima: Tesis Doctoral. Universidad Privada de Lima.

Chiavenato, I. (2007). Administración de recursos humanos. México : McGraw-hill.

Flores Aguilar, R. (2015). Gestión de la responsabilidad social univeristaria y la calidad acdémica en las universidades del departamento de Puno año 2013. Puno - Perú: Universidad Andina Néstor Cáceres Velásquez.

Gaete, R. (2012). Responsabilidad social universitaria: una nueva mirada a la relación de la universidad con la sociedad desde la perspectiva de las partes interesadas. Un estudio de caso. España: Tesis Doctoral. Universidad de Valladolid.

Gil, F. (2013). La responsabilidad social universitaria desde la perspectiva ambiental: universidad y desarrollo sustentable. México: Tesis Doctoral. Universidad Autónoma.

Hernández, R. y. (2019). Metodología de la investigación: Las rutas cuantitativa, cualitativa y mixta. México: McGraw Hill.

Landeau, R. (2007). Elaboración de trabajos de investigación. Venezuela: Alfa.

Miranda, E. H. (2019). Responsabilidad social universitaria y satisfaccional laboral del personal administrativo en una univerisdad pública de Huancayo (Perú). Huancayo-Perú: Centro de Investigación FS-UNCP.

Oseda, D. H. (2018). Estadística aplicada a la investigación con SPSS. Huancayo - Perú: Soluciones y Gráficas SAC.

Reyna, G. (2018). Percepción de las autoridades sobre la responsabilidad social universitaria en la Universidad Nacional del Centro del Perú 2018. Trujillo - Perú: Tesis Doctoral. Universidad César Vallejo. 
Rivera, H. y. (2011). La organziación de los satkeholders y la responsabilidda social. BogotáColombia: Universidad del Rosario. .

Shankman, N. (1999). Reframing the debate between agency and stakeholder theories of the firm. Journal of Business Ethics, s/n.

Sierra, R. (2004). Tipos de investigación. Barcelona, España: Paraninfo S.A.

Tamayo, M. (1995). El proceso de investigación cientifica. México: Limusa S.A.

Toche, E. (Octubre de 2013). Responsabilidad Social en el Perú. Percepción de la Empresa y $O N G, 48$.

Universidad Construye País. (30 de abril del 2018 de 2002,a). La Universidad Construye País. Obtenido de Universidad Católica de Valparaiso: http://www.bibliotecavirtual.info/wp

Vaca, R. (2007). Analísis de la responsabilidad social corporativa desde tres enfoques: stakeholders, capital intelectual y teoría institucional. Logroño - España: Universidad de la Rioja.

Valerrama, S. (2019). Pasos para elaborar proyectos de investigación científica: Cuantitativa, Cualitativa y Mixta. Lima - Perú: San Marcos.

Vallaeys, F. (2006). Breve marco teórico de la Responsabilidad social universitaria. Lima: Pontificia Universidad Católica del Perú.

Vallaeys, F. (2008). Responsabilidad social univrsitaria: una nueva filosofia de gestión ética e inteligencia para las universidades. Educación superior y sociedad, 193-220.

Vallaeys, F. D. (2009). Responsabilidad social universitaria. Manual de primeros pasos. México D.G.: McGraw-Hill.

Vargas, M. (2017). Responsabilidad social universitaria desde la percepción del estudiante de la escuela profesional de administración de la Universidad Nacional de San Agustín de Arequipa -2016. Arequipa: Tesis Doctoral. Universidad Nacional de San Agustín. 